\title{
Neurotrophic factors and Parkinson's disease
}

\begin{abstract}
Neurotrophins play a major role in adult neuronal survival, maintenance and regeneration. Alterations in their levels have been implicated in various neurodegenerative disorders, including Parkinson's disease (PD). It has been reported that, the pathophysiology of PD progress is essentially depends on various striatal signaling cascade, which consists handful of neurotrophic factors namely, cerebral dopamine neurotrophic factor (CDNF), glial derived neurotrophic factor (GDNF), mesencephalic astrocyte-derived neurotrophic factor (MANF), brain derived neurotrophic factor (BDNF), nerve growth factor (NGF). Although, the exact pathophysiology of PD is remained elusive however, the loss of dopaminergic neurons and dopamine deficiency has considered as a major consequence for the movement disability as seen in PD. It has been proposed that loss of dopaminergic neurons in PD may be conducted by inadequate neurotrophic activity which leads to neuronal apoptosis. In addition, stimulation of neurotrophic factors in the striatal brain region has been reported to be beneficial in experimental models of PD. In the current review we have detailed out the neurotrophic factors and their role in pathogenesis of Parkinson's disease.
\end{abstract}

Keywords: Parkinson's disease - Neurotrophic factors - NGF - BDNF - GDNF - MANF - CDNF Movement disorders

Submitted Date: 08 February 2018; Accepted Date: 24 February 2018; Published Date: 03 March 2018

\section{Abbreviations}

PD: Parkinson's Disease, NGF: Nerve Growth Factor, BDNF: Brain Derived Neurotrophic Factor, NT: Neurotrophins, GDNF: Glial Derived Neurotrophic Factor, CNTF: Ciliary Neurotrophic Factor, Trk: Tyrosine receptor kinase; p75 ${ }^{\text {NTR: }}$ pan-75 Neurotrophic Receptor; NTFs: Neurotrophic Factors.

\section{Introduction}

The neurotrophins are basically defined as growth factors, in which there main functions are to regulate distinction and growths while the evolution of nervous system. The study of neurotrophins led to the hypothesis that neurons should have bonding with each other for specific survival factors $[1,2]$. A similitude imagines would be growing two plants in a pot. These two plants compete with each other for the limited amount of nutrients in the soil, and the plant better capable to take up nutrients would be more likely to survive. The neurotrophic factor hypothesis proposed that presence or absence of neurotrophins determines whether a given neuron will live or die [3]. There are so many affirmations that NTFs and their families work in synchronization and affect each other. Neurotrophins family comprises of Ciliary neurotrophic factors (CNTF), Brain-derived neurotrophic factor (BDNF), Neurotrophin 4 (NT4), Neurotrophin 3 (NT3), Nerve growth factor (NGF) and Glial-derived neurotrophic factors (GDNF). There are two more factors in neurotrophins family i.e. Mesencephalic astrocyte-derived neurotrophic factor (MANF) and Cerebral
Rajat Bhardwaj' and Rahul Deshmukh2*

${ }^{1}$ Neuropharmacology Division, Department of Pharmacology, ISF College of Pharmacy, Moga- 142001, Punjab, India.

${ }^{2}$ Department of Pharmaceutical Sciences \& Technology,

Maharaja Ranjit Singh Punjab Technical University, Bathinda -151001, Punjab, India

*Author for correspondence: E-mail: 9rajatbhardwaj9@gmail.com; drrahuld09@gmail.com;

rahuldeshmukh@mrsptu.ac.in 
dopamine neurotrophic factor (CDNF; official name by HUGO gene nomenclature committee; previous name conserved dopamine NTF) which involve in development of protein family responding neurotrophic activities [4-6]. MANF reported as founding member of the family and identified from the culture medium of rat Type-1 astrocyte ventral mesencephalic cell line 1 (VMCL1) based on its supporting and developing activities on cultured embroynic dopaminergic neurons. CDNF was first identified by bioinformatics and later biochemically characterized. Mature CDNF protein consists of 161 amino acid residues (pre CDNF is 187 amino acids long), and is a vertebrate specific paralog of MANF. CDNF and MANF encoding genes are highly conserved in evolution. In order to elicit a survival response, each of them binds to one member of the tyrosine receptor kinase (Trk) family: NGF to TrkA, BDNF and NT4 to TrkB, and NT3 to TrkC. After binding to pan-75 neurotrophin receptor (p75NTR), apoptotic pathway imitated and responds to each of the neurotrophins. It has been hypothesized that due to improper activity of trophic factors there will be neuronal apoptosis which may leads to loss of dopaminergic neurons in Parkinson's disease (PD). However, it was not reported whether neurons death or decrease in level of neurotrophins involved in PD or any other neuronal loss accompanied to disease in humans [7]. Regardless of the causes of PD; Neurotrophins, by promoting DA neuronal growth and function and by interfering with neurotoxic processes, could be of therapeutic value.

\section{Neurotrophins}

Neurotrophins are diffusible peptides secreted from neurons and neuron-supporting cells. They serve as growth factors for the development, maintenance, repair, and survival of specific neuronal populations. During development, NTFs promote neuronal survival, stimulate axonal growth, and play a key role in the construction of the normal synaptic network [8]. Therefore, any alterations in their local synthesis transport or signaling (e.g. binding, internalization, receptor synthesis etc.) due to local damage, aging, mutation, or polymorphism could adversely affect neuronal survival and lead to neuronal death. Indeed studies shows that alteration of neurotrophins for selective neuronal population might correlate with the neurodegeneration. Recently, researchers have directed their attention to the identification of those conditions promoting human neuronal survival and repair in neurodegenerative diseases.

\section{Structure of neurotrophins}

The neurotrophins are produced as precursor proteins, which are cleaved at dibasic amino acids to produce a mature form of 118-130 amino acids. Neurotrophin is a noncovalent dimer of $13 \mathrm{kDa}$ polypeptide chains. Each protomer is bound together by three disulfide bridges arranged in a highly characteristic cysteineknot motif, a feature common to all members of the neurotrophin family. The NTs protomer consists of two pairs of antiparallel $\beta$-strands that form an elongated dimer interface. The NT dimer interface is held together by noncovalent interactions, largely comprised of hydrophobic interactions between aromatic residues.

\section{Discoveries of neurotrophins}

The era of growth factors research began fifty years ago with discovery of different neurotrophic factors with their physiological role and potential clinical application (Figure 1).

\section{Types}

The types of neurotrophins consists of cerebral dopamine neurotrophic factors (CDNF), mesencephalic astrocyte-derived neurotrophic factors (MANF) Brainderived neurotrophic factor (BDNF), Neurotrophin 4 (NT4), Neurotrophin 3 (NT3), Nerve growth factor (NGF) and Glial-derived neurotrophic factors (GDNF) (Table 1).

\section{Signaling pathways}

The activity of neurotrophins is through Trk receptors. Neurotrophins at physiological conditions have specificity with Trk receptors. There are different type of Trk receptors which binds with NTs i.e.; TrkA binds with NGF, TrkB binds with both BDNF and NT-4 and TrkC binds with NT-3 [9]. The expression of these receptors is having similarities with known affectability of neurons to particular neurotrophins. After attaching on the surface of NT, the Trk receptor tyrosine kinase activation initiated and the most basic substrate of this action regulated by the receptor itself. The receptor turns out to be quickly autophosphorylated, and this is essential for the receptor activity. The receptor autophosphorylation shapes the docking destinations for the association of downstream signaling molecules. Most proteins that are having affinity for binding with phosphorylated tyrosine can be categorized as one of two gatherings. The most well-known phospho tyrosine binding is the src-homology area 2, or SH-2 domain. SH-2 areas are ordinarily distinguished in view of their homology to other SH-2 domain- containing proteins. Some 


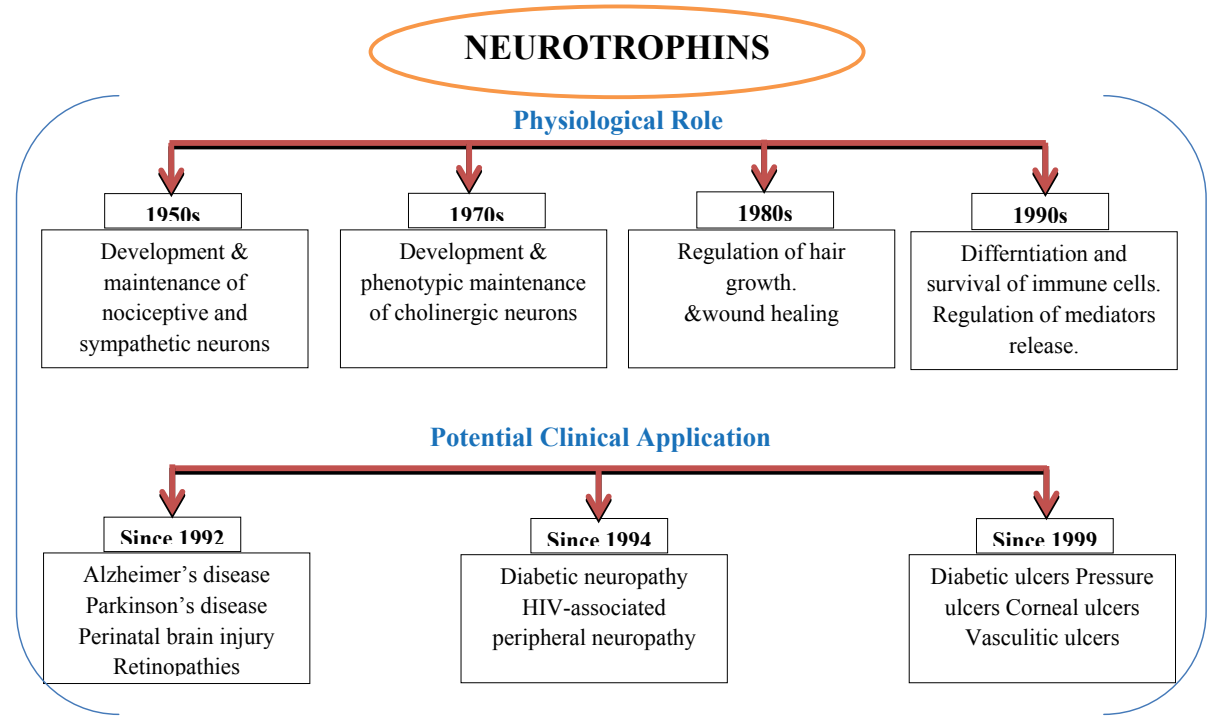

Figure 1: Discoveries of neurotrophins

of these have been indicated specifically to have affinity for phosphorylated tyrosine in the proper amino acid context. The SH-2 domain-containing proteins additionally frequently interact with proteins containing, a src-homology space 3 , or $\mathrm{SH}$ 3. After these flagging proteins tie to the activated autophosphorylated receptor, where they end up becoming activated [10]. The components by which they are enacted were as yet obscure. Frequently they, excessively progress toward becoming phosphorylated on tyrosine residues. Moreover, their adherence to the membrane or into signaling complexes assumes a crucial contribution in the initiation of specific activity. Actuation of each of these NT-signaling proteins triggers particular downstream cascades of target enzymes and other biological impacts [11]. In spite of the fact that there is incredible assorted variety of neurotrophic factor receptors, they appear to trigger just a couple of very much conserved sorts of downstream signaling pathways. Among the best described of the pathways consists the Ras/ extracellular signal regulated kinase (ERK) pathway, the phosphotidylinositol-3'- OH-kinase (PI3K) pathway, and the phospholipase C-(PLC-) pathway. Furthermore, particular tyrosine phosphates are activated that regulate these responses and may contain pathway-actuating properties of their own. There is likewise confirmation of cross-talk between G-protein-coupled receptor flagging pathways and the NT cascades. A wide range of communications happen between the $G$ protein receptor- coupled second messenger pathways and the Trk signaling pathways (Figure 2) [12].

\section{P75 Neurotrophin receptor -mediated signaling pathways}

The p75NTR belongs to the tumor necrosis factor receptor family and was the first identified neurotrophin receptor. The $\mathrm{p} 75$ neurotrophin receptor binds all of the neurotrophins equally, with relatively single- affinity compared with Trk binding [13]. Expression of p 75 can increase the affinity of TrkA for NGF and can enhance its specificity for cognate Neurotrophins [14]. Although, it does nothave a catalytic intracellular tyrosine kinase domain, it is capable of mediating the neurotrophin signals. This happens both by its modulating TrkA signaling and independently of Trk. The ligand binding of p75NTR increases the high-affinity TrkA binding sites enhances TrkA autophosphorylation and selectivity for neurotrophin ligands [15]. The Trk-independent pathway of p75NTR increases intracellular ceramide levels and further activates NFkB transcription factor and JNK kinase. Conversely, TrkA activation inhibits p75NTR-mediated signaling, but the mechanism of this inhibition is unclear. One established function of p75 is to promote cell death [16]. P75 receptor is known to bind the unprocessed pro neurotrophins with ligand engagement resulting in dramatically different consequences, such as apoptosis, than those that follow ligand engagement of Trk receptors [17].

Several signaling pathways are activated following neurotrophin binding to p75NTR. These are mediated through binding p75NTR of several adaptor proteins, including Traf6, neurotrophin receptor-interacting factor (NRIF), melanoma-associated antigen (MAGE), neurotrophin receptor p75 interacting MAGE 
Table 1: Types of neurotrophins

\section{Neurotrophins in brain function}

It is the first discovered type of Neurotrophins family. NGF was discovered 50 years ago as a molecule for differentiation and survival of sensory and sympathetic nervous system. In the central nervous system (CNS),

Nerve Growth Factor

Brain Derived Neurotrophic Factor

Neurotrophin 4/5

\section{Glial cell-}

derived neurotrophic factor

Mesencephelic astrocytederived neurotrophic factors

Cerebral dopamine neurotrophic factors the greatest amount of NGF is produced in the cortex, the hippocampus and in the pituitary gland; although important quantities of this neurotrophin are also produced in some other areas, including the basal ganglia, thalamus, spinal cord and in the retina. The NGF plays a major role in the survival and function of cholinergic neurons of the basal forebrain complex (BFC), such functions include attention, arousal, motivation, memory and consciousness.

In 1982, BDNF the second member of the "neurotrophic" family of neurotrophic factors, was shown to promote survival of a subpopulation of dorsal root ganglion neurons, and subsequently purified from pig brain. BDNF has growth and survival actions on a variety of neurons, including dorsal root ganglion cells and hippocampal and cortical neurons. The expression of BDNF gene in physiologic regulation may be very important in the development of the brain. In neuropsychiatry, BDNF involved with several disorders, such as substance related disorders, eating disorders, mood disorders, schizophrenia, pain modulation and neurodegeneration. It appears to have more widespread neurotrophic effects influencing a variety of non-cholinergic systems including dopamine perikarya within the substantianigra. Co-expression of BDNF and its receptor suggests that, in addition to its role as a classical target-derived neurotrophic factor, the neurotrophin may also be effective locally via autocrine or paracrine mechanisms. Therefore, BDNF which specifically prevents degeneration and increases the functional activity of dopaminergic neurons, is of substantial interest for the development of new therapeutic strategies as a neuroprotection therapy for PD. BDNF also supports role in the learning and memory and there is a good correlation between BDNF and mRNA expression in learning and memory tests.

Neurotrophin 3 is the third member of the neurotrophin family. Neurons responsive to NT-3 include cells in both the peripheral and central nervous systems. Among responsive peripheral cells are neurons of the dorsal root ganglia and no dose ganglion and possibly the sympathetic ganglia. In the central nervous system, NT-3responsive cells include neurons of the trigeminal mesencephalic nucleus, hippocampus, and dopaminergic and GABAergic cells in the ventral mesencephalon. Motor neurons of the spinal cord also respond. In the peripheral nervous system, NT-3 mRNA is found in muscle. In the central nervous system, high levels of NT-3 mRNA are found in the hippocampus and cerebellum.

Neurotrophin $\mathbf{4 / 5}$ is the most recently discovered member of the neurotrophin gene family. Cells in the peripheral nervous system that are responsive to NT-4/5 include trigeminal, dorsal root ganglion, jugular, sympathetic, and no dose ganglion neurons(Rocamoraa and Arenasb 1996). In the central nervous system, hippocampal neurons and dopaminergic and GABAergic neurons of the ventral mesencephalon respond, as do motor neurons. Messenger RNA of NT-4/5 is found in muscle and other peripheral tissues and in the central nervous system(Ip and Yancopoulos 1994). The highest amounts in the central nervous system were found in pons or medulla, hypothalamus, thalamus, and cerebellum. Neurotrophin $4 / 5$ is known to activate the trkB receptor.

GDNF was the first identified member of a family of factors, which includes neurturin (NTN), enovin and artemin (ART), all of which are distant members of the transforming growth factor-beta (TGF-beta) superfamily(Nutt, Burchiel et al. 2003). GDNF was found in a classical neuronal survival approach used in the search for secreted neurotrophic factors(Lin, Doherty et al. 1993). GDNF was new potent neurotrophic molecule was identified because of the ability of the conditioned media of glial cell line cultures to promote the survival of dopaminergic neurons. In addition, GDNF family proteins are potent survival factors for several populations of central and peripheral neurons.

MANF is new neurotrophic factors which Selectively promotes the survival of dopaminergic neurons of the ventral mid-brain. Modulates GABAergic transmission to the dopaminergic neurons of the substantia nigra. Enhances spontaneous, as well as evoked, GABAergic inhibitory postsynaptic currents in dopaminergic neurons. Inhibits cell proliferation and endoplasmic reticulum (ER) stress-induced cell death.

CDNF: (official symbol of HUGO gene nomenclature committee. Previously known as conserved dopamine neurotrophic factors). CDNF is secretory neurotrophic factor, consisting of two domains. Asaposin like $-\mathrm{N}$ terminal domain and a presumably unstructured carboxy- (C) terminal with intradomain cysteine bridge in a CXXC motif. Though the receptor of CDNF, is unclear yet, the N-terminals domain of CDNF interacts with lipids membranes as the saposin like protein do (Bruhn 2005).

homologue (NRAGE), Schwann cell factor 1 (SC1), RhoGDI and other proteins [18]. One major pathway activated by p75NTR engagement by neurotrophins is the Jun kinase signaling cascade. Signaling through this cascade results in activation of $\mathrm{p} 53$ and apoptosis. In addition, the Jun kinase cascade induces expression of Fas ligand in neuronal cells, promoting apoptosis through activation of the Fas receptor (Figure 3) [19].
Binding specificity of neurotrophins: The binding of neurotrophin ligands to their respective cellular receptors initiates intracellular signals essential for the growth and survival of neurons (Table 2).

\section{Neurotrophins and Parkinson's disease}

Parkinson's disease is an idiopathic neurodegenerative disorder which affects more than million people 


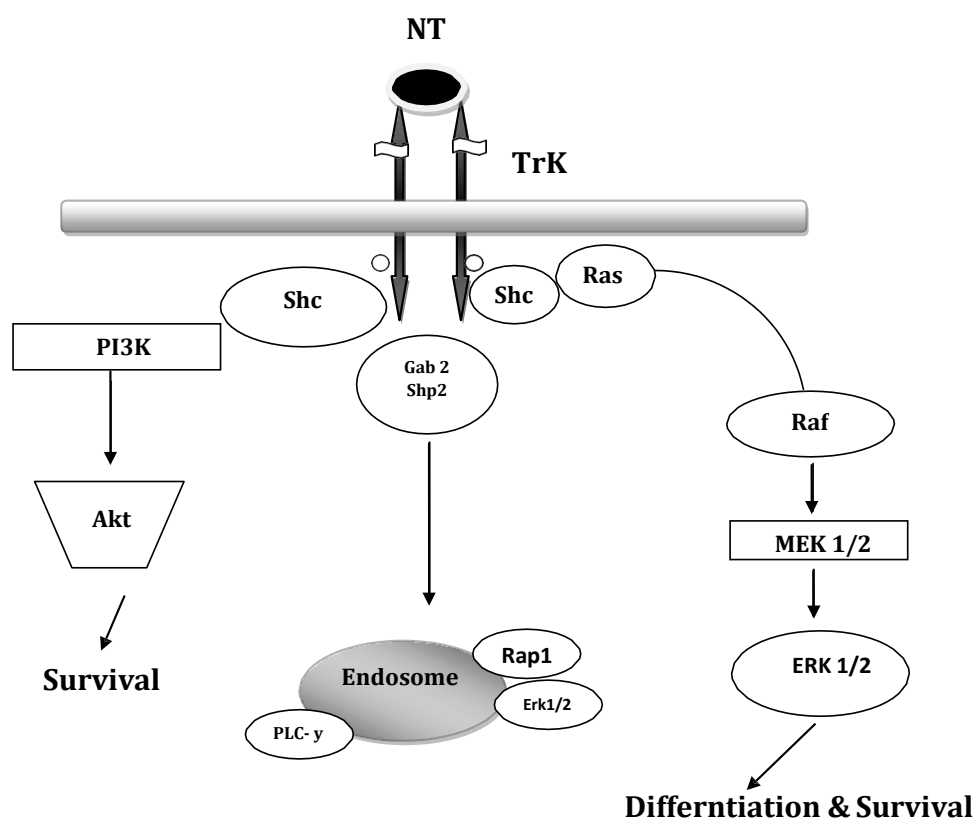

Figure 2: Trk Signaling pathway: The key components of the pathway are Ras, Shc, Gab2. Neurotrophin binds to Trk receptor and phosphorylates Shc domain which in turn activates Ras Signaling and PI3K/AKT pro survival pathway (MEK: mitogen-activated protein kinase; ERK: extracellular regulated protein kinase; RAF: rapidly accelerated fibrosarcoma protein; RAS: proto-oncogene serine/threonine-protein kinase; PI3K: phosphotidylinositol-3'-OH-kinase; PLC: phospholipase C; GAB: Grb- associated binding scaffold protein; RAP: Ras related protein; SHC: src homology)

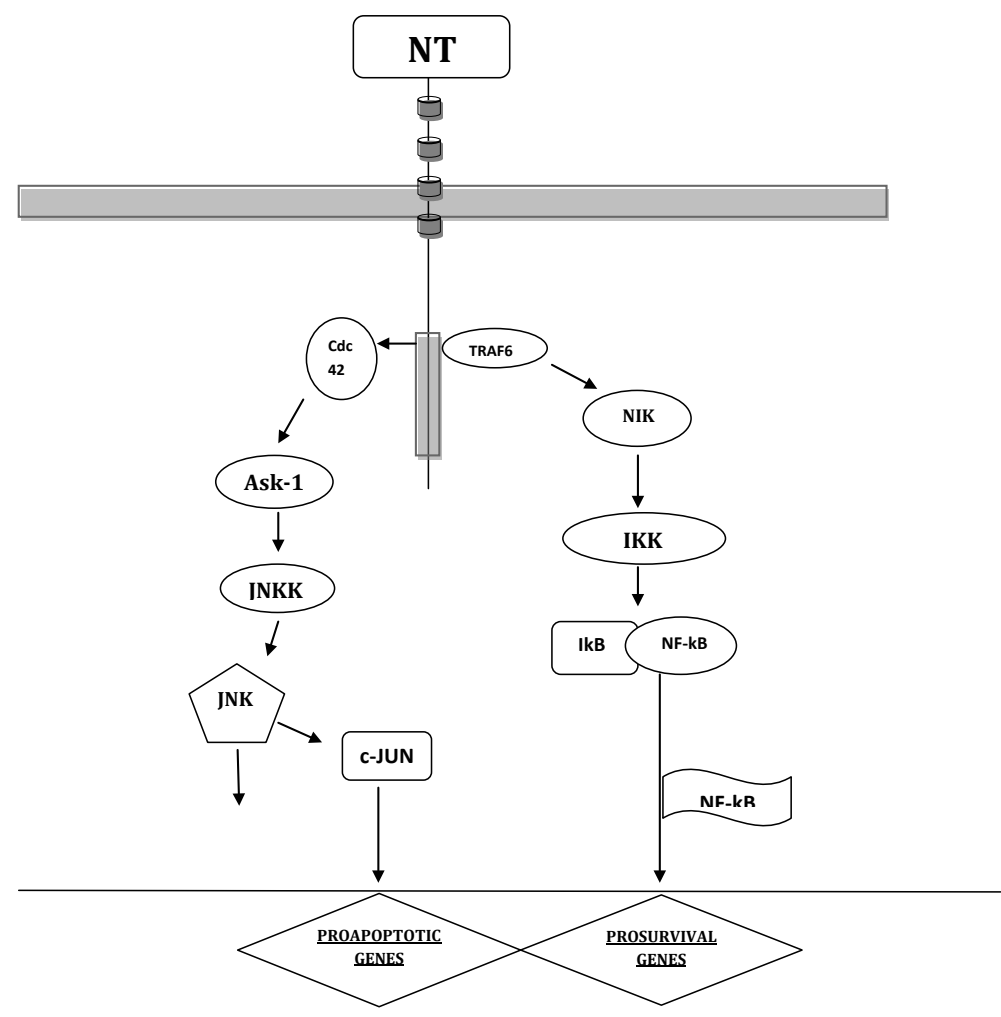

Figure 3: $p 75^{\text {NTR }}$ Signaling pathway: The key components of the pathway NF-kB and JUN kinase pathway. When p75 binds to neurotrophin, NF-kB gets activated and results in transcription of multiple genes that promotes neuronal survival and activation of Jun kinase pathway similarly controls activation of several genes which promotes neuronal apoptosis and modulates Rho activity (NFkB: nuclear factor kappa-light-chain-enhancer of activated B cells; JNK: Jun N terminal kinase; TRAF6: tumour necrosis factor receptor associated factor 6; NIK: NFkB inducing kinase; IKK: inhibitor of nuclear kappa-B kinase) 


\begin{tabular}{|c|c|c|c|}
\hline Receptor & Preferred Ligand & Non-Preferred Ligand & Non-Ligand \\
\hline TrkA & NGF & NT-3, NT-4 & BDNF \\
\hline TrkB & BDNF, NT-4 & NT-3 & NGF \\
\hline TrkC & NT-3 & None & NGF, BDNF \& NT-4 \\
\hline P75 & NGF, BDNF, NT-3, NT-4 & None & None \\
\hline
\end{tabular}

worldwide. Aging is major cause of PD as according to rising in age of common population, the prevalence of PD will increase in future [20]. There are motor and non-motor symptoms which are sign of PD, motor symptoms are rigidity, tremor, bradykinesia and postural abnormalities and non-motor are cognitive impairment, anxiety, fatigue and depression but there is still no biological marker that confirms the diagnosis for PD. PD is signalized by the progressive death of selected heterogeneous bundles of neurons, including the neuromelanin-laden dopaminergic neurons of the SNpc [21]. Neuronal loss founds to be more in the ventrolateral tier followed by the medial ventral tier and dorsal tier within the $\mathrm{SNpc}$, not all dopamine neurons prediction are equally receptive [22]. It results in a regional loss of striatal dopamine, most prominently in the dorsal and intermediate subdivisions of the putamen, a process that is believed to account for akinesia and rigidity. Despite the great research efforts of the last decade, the etiopathogenesis of PD is still unknown. L-dopa and DA agonists are currently used to alleviate symptoms of PD, but most treated patients still develop a progressive functional disability that severely affects their quality of life [23]. The mechanisms responsible for cell death in PD are largely unknown. Increasing evidence suggests that neuronal death in the SNpc may be apoptotic and the neurotrophins are well reported to overcome this neuronal death [24].

Neurotrophins have clinical role in neuronal functioning, and is not necessary that neurodegenerative and other related disorder are due to altered neurotrophic factors and with change in their receptor expression [25]. As, increased expression of NTFs have neuroprotective role against brain damage to protect neurons against oxidative stress, excitotoxicity and apoptosis [26].

It has been reported that NGF family and their receptors are confined in melanin-positive neurons of Substantia Nigra pars compata (SNpc) [27]. Further, it was reported that, in PD pathology changes or decrease in the BDNF and NGF levels are quite common, which is unlike the changes in stain of NT3 or NT4/5 in PD [28]. Intriguingly, watched loss of DA neurons in PD has been found to be associated with the lack of trophic factors, which assists neuronal fate towards apoptosis. Notwithstanding, equivocal reports on neuronal loss is principally points towards apoptotic processes in PD; however, the role of decreased trophic factors mediates such apoptotic process, are less studied in PD [29]. NGF family of trophic factors is abundant in dopaminergic neurons and their release is associated with BDNF function [30]. Though, in vivo studies showed the insufficiency of BDNF did not overcome nigral dopamine loss [31]. Further, a recent study has demonstrated that, 6-OHDA administration and then BDNF and NTF4/5 treatment could increase dopamine metabolism. Another study showed that, BDNF not only replenishes the DA level but also rescues the GABAergic neurons after ibotenic-induced toxicity at striatum/pallidus. This result also supports the notion of glutamate normalization by BDNF. It is also reported that, BDNF promotes protective attributes against MPTP-induced parkinsonian toxicity in mesencephalic DA neurons of rodent and primate PD models. Moreover, administration of NGFs secures $\mathrm{TH}$ positives neurons from MPTP induced toxicity in animal model of PD [32].

In recent research some of the researchers observed that GDNF family elevates the survival and differentiation of dopaminergic neurons in midbrain 6-OHDA induced rat models of PD [33]. It was found by the biochemical and behavioral parameters like; route of administration, place of injection, age of rats and method of delivery into the brain and after these studies it was concluded that GDNF ameliorated the behavioral and biochemical parameters of 6-OHDA model of PD [34]. The main evidence of the 6-OHDA induced model associated with adeno-associated viral expressing GDNF state that viral delivery of a GDNF gene with couple of cell lines rescued and protected dopamine neurons. Neurturin (NTN) delivery in the SNpc also shown to be effective in protection of nigral neurons in 6-OHDA induced model. In the adult rat striatum of 6-OHDA lesions model, GDNF administration showed most protective effect to recover the dopaminergic neurons than administration of NTN [35]. This concludes that in the striatum, NTN 
is less protective then GDNF. Another member of GDNF family which is known as Persephin (PSP) was also shown to prevent the loss of dopamine neurons and improve behavioral impairments of 6-OHDA lesioned rats. When GDNF injected in the SNpc or into striatum before and after MPTP administration in rats and monkeys potently protected the dopamine system, as indicated by the number of mesencephalic dopamine nerve cell bodies, density of dopamine nerve terminals, levels of dopamine and motor behavior. In several studies it has been observed that adenoviral, adeno-associated viral, or lentiviral vectors expressing GDNF reported dopamine neurons protection against MPTP and 6-OHDA toxin in rats and monkeys [36]. A combined GDNF and BDNF treatment was also found to have a protective effect of nigral tissue.

CDNF and MANF are expressed in the developing nigro-striatal system of the mouse at $\mathrm{P} 1$ and $\mathrm{P} 10$, that is around the two postnatal PCD peaks of midbrain dopaminergic neurons at $\mathrm{P} 2$ and $\mathrm{P} 14$, suggesting that CDNF and MANF may have a role in the development of midbrain dopaminergic system. In the adult mouse, MANF protein was also localized in TH positive dopaminergic neurons in the Substantia Nigra, whether MANF or CDNF function as targetderived NTFs for midbrain dopaminergic neurons is currently unknown. Future studies on CDNF and MANF-deficient mice should reveal the developmental role of these new NTFs. The widespread expression of CDNF and MANF suggests that endogenous CDNF and MANF secreted by other sources than the striatal target may affect maturation of rodent dopaminergic system in vivo [37]. Whether CDNF and MANF affect PNS neurons or other neuronal types in addition to dopaminergic neurons and cortical neurons in the CNS in vivo is unknown. In vitro, CDNF protein was unable to promote the survival of embryonic motor neurons or dorsal root ganglion (DRG) neurons, or early postnatal sympathetic cervical ganglion (SCG) neurons differently from GDNF that supports the survival of different neuronal populations including motoneurons and enteric, sensory, parasympathetic, and sympathetic neurons. Similar to the results obtained with CDNF were also reported for the MANF protein, although MANF showed a small survival promoting effect on DRG neurons [38].

Cdnf and Manf are expressed in the developing nigrostriatal system of the mouse at P1 and P10 (Lindholm et al., 2007, 2008), that is around the two postnatal PCD peaks of midbrain dopaminergic neu- rons at $\mathrm{P} 2$ and P14 (Burke, 2004), suggesting that CDNF and MANF may have a role in the develop- ment of midbrain dopaminergic system. In the adult mouse, MANF protein was also localized in $\mathrm{TH}$ - positive dopaminergic neurons in the SN (Lindholm et al., 2008). Whether MANF or CDNF function as target-derived NTFs for midbrain dopaminergic neu- rons is currently unknown. Future studies on CDNF- and MANFdeficient mice should reveal the develop- mental role of these new NTFs. The widespread expression of CDNF and MANF (Lindholm et al., 2007, 2008) suggests that endogenous CDNF and MANF secreted by other sources than the striatal tar- get may affect maturation of rodent dopaminergic system in vivo Cdnf and Manf are expressed in the developing nigro-striatal system of the mouse at P1 and P10 (Lindholm et al., 2007, 2008), that is around the two postnatal PCD peaks of midbrain dopaminergic neu- rons at P2 and P14 (Burke, 2004), suggesting that CDNF and MANF may have a role in the develop- ment of midbrain dopaminergic system. In the adult mouse, MANF protein was also localized in $\mathrm{TH}$ - positive dopaminergic neurons in the SN (Lindholm et al., 2008). Whether MANF or CDNF function as target-derived NTFs for midbrain dopaminergic neu- rons is currently unknown. Future studies on CDNF- and MANFdeficient mice should reveal the develop- mental role of these new NTFs. The widespread expression of CDNF and MANF (Lindholm et al., 2007, 2008) suggests that endogenous CDNF and MANF secreted by other sources than the striatal tar- get may affect maturation of rodent dopaminergic system in vivo

\section{Neurotrophins Crosstalk with Neurobi- ology of PD \\ Dopaminergic signaling}

Dopamine is one of the main monoaminergic neurotransmitters in the central nervous system. The mesodiencephalic dopaminergic (mDA) neuronsresponsible for the major source of dopamine in the brain as, PD is a movement disorder characterized by a progressive loss of midbrain dopaminergic (DA) neurons of the SNpc. The cause of degeneration of DA neurons in patients with PD is not completely understood, but mitochondrial dysfunction, oxidative stress, protein aggregations, and reduced neurotrophic signaling are commonly observed in PD patients [39]. This multiple-hit hypothesis states that these assembled alterations are finally leading to the collapse of the protein network essential for DA cell survival [40]. Recent, discoveries shows that mice that have genetically lost the GDNF receptor, the receptor tyrosine kinase 
RET showed an age- and cell type-specific loss of SNpc DA neurons. But why the DA system depends on neurotrophic GDNF/RETS signaling and which downstream signaling cascades are used for their beneficial effect is still unknown. In recent studies, it states that RET and DJ-1, a protein mutated in familial forms of $\mathrm{PD}$, are required to overcome DA cell body maintenance through the RAS/MAPK pathway. The PD associated gene PARK2 which encodes the protein parkin, an E3 ubiquitin protein ligase important for mitochondrial probity and quality control [41]. Since the parkin gene and RET have been shown to function in the protein network altered in patients with PD and parkin influences intracellular Signaling cascades of other receptor tyrosine kinase [42]. Interestingly, elevated parkin and GDNF/RET signaling can prevent mitochondrial dysfunction caused by either RET or parkin deficiency. As, parkin and RET together protect mitochondrial integrity and function through the phosphoinositide-3-kinase/NF- $\mathrm{BB}$ (PI3K/NF- $\mathrm{KB}$ ) pathway, which can prevent $\mathrm{DA}$ neuron degeneration [43]. To attain more detail of parkin and Ret in DA neurons, there is a two models, the first mouse model was parkin and Ret double-deficient mice which showed an accelerated dopaminergic cell loss specifically in the substantia nigra and dopaminergic fiber loss in the striatum compared to no alterations in parkin- and a moderate degeneration in Ret-deficient mice. The double deficient mice also showed reduced dopamine levels, increased anxiety and reduced ATP and mitochondrial complex I activity as also reported for PD patients. The second mouse model was mice over expressing human parkin in a Ret-deficient background which did not show the age related dopaminergic system degeneration observed in Ret-deficient mice [44]. This suggests that enhanced parkin signaling is not only protective against toxin- and stress induced dopaminergic system degeneration, but also against neurotrophic deprivation induced by Ret loss [45].

\section{Non-dopaminergic signaling}

Although, dopaminergic degeneration is a prominent mechanism of PD pathology, however, the exact cause of this degeneration remained elusive. Moreover, current drug therapy aiming to improve dopaminergic signaling did not completely restore motor functions $\&$ is associated with other non-motor symptoms such as dyskinesia, anxiety, depression, fatigue, sleep disturbance, or sensory symptoms [46]. In Parkinson's disease, when the level of dopamine decreases, there is also the decrease in another neurotransmitter, acetylcholine since the motor function of the striatum is dependent on the equilibrium between dopamine and acetylcholine. This further disruption results in imbalance of neurotransmitters which contributes to disease progression. Dysregulation in the other neurotransmitters, norepinephrine, serotonin, and gamma amino butyric (GABA), may further contribute to secondary symptoms related to PD [47]. Variety of other factors has been considered to be liable for dopaminergic neuronal degeneration such as imbalance between GABAergic \& Glutamatergic signaling. Deficiency of GABAergic Signaling has been implicated in the development of motor dysfunction whereas up regulation in glutamatergic signaling has been well known to cause excitotoxic neuronal cell death in the striatal region of the brain. In addition increased oxidative stress, neuroinflammation etc. has also been implicated in striatal degeneration as seen in PD [48].

\section{Conclusion}

BDNF, NGF, GDNF, MANF and CDNF have been associated with neuroprotection and neuroplasticity. Above data suggests that neurotrophins play an important rolein neural development, neurotransmission and improvement of dopaminergic neuronal loss in Parkinson's disease, leading to betterment of motor functions in PD patients. Therefore, it can be concluded that neurotrophins would be helpful in therapeutic in the management of motor dysfunction and associated comorbidities as seen in PD.

\section{Acknowledgement}

The authors are thankful to the Science and Engineering Board (SERB), Department of Science and Technology, Govt. of India, New Delhi, for providing financial assistance under the Fast Track Scheme (DST-SERBFTYS) (SB/YS/LS-111/2013) to Dr. Rahul Deshmukh. 


\section{References}

1. Lewin GR, Barde YA. Physiology of the neurotrophins. Ann rev neurosci19: 289-317 (1996).

2. Kimpinski K, Campenot RB Mearow K. Effects of the neurotrophins nerve growth factor, neurotrophin-3, and brainderived neurotrophic factor (BDNF) on neurite growth from adult sensory neurons in compartmented cultures. $J$ neurobiol 33: 395-410 (1997).

3. Zhang Y, Zhang C, Cheng Z, et al. Serum levels of brain-derived neurotrophic factor and clinical efficacy of mirtazapine in geriatric patients with major depression. Biomed Res 26: (2015).

4. Petrova PS, Raibekas A, Pevsner J. MANF. J Mol Neurosci 20: 173-187 (2003).

5. Lindholm P, Voutilainen MH, Lauren J. Novel neurotrophic factor CDNF protects and rescues midbrain dopamine neurons in vivo. Nature 448: 73-77.

6. Voutilainen MH, Back S, Porsti E. Mesencephalic astrocytederived neurotrophic factor is neurorestorative in rat model of Parkinson's disease. J Neurosci 29: 9651-9659 (2009).

7. Weissmiller AM, Wu C. Current advances in using neurotrophic factors to treat neurodegenerative disorders. Transl Neurodegener 1: 1 (2012).

8. Wu L, Liu YM. Factors associated with Parkinson's disease patients with hyposmia in Chinese han population: a casecontrol study. Biomed Res 27: (2016).

9. Bafico A, Aaronson SA. Classification of growth factors and their receptors. Holland-Frei Cancer Medicine. Hamilton (ON): $B C$ Decker 6: 2003.

10. Krieglstein K. Neurotrophic Factors. Encycl Mol Pharmacol 843845 (2008).

11. Jieming Z. Construction of nerve growth factor loop 4-containing polypeptides for facilitated gene transfer to neurons (Doctoral dissertation: 2005).

12. Skaper SD. The biology of neurotrophins, signalling pathways, and functional peptide mimetics of neurotrophins and their receptors. CNS \& Neurol Dis-Drug Targets (Formerly Curr Drug Targets-CNS \& Neurol Diss) 7: 46-62 (2008).

13. Chao MV, Bothwell M. Neurotrophins: to cleave or not to cleave. Neuron 33: 9-12 (2002).

14. Huang EJ, Reichardt LF. Trk receptors: roles in neuronal signal transduction*. Ann Rev Biochem 72: 609-642 (2003).

15. Schweigreiter R. The dual nature of neurotrophins. Bioessays 28: 583-594 (2006).

16. Hempstead BL. The many faces of p75 NTR. Curr opin neurobiol 12: 260-267 (2002).

17. Martins IJ. Anti-aging genes improve appetite regulation and reverse cell senescence and apoptosis in global populations. Adv in Aging Res 5: 9-26 (2016).

18. Nykjaer A, Willnow TE, Petersen CM. p75 NTR-live or let die. Curr opin neurobiol 15: 49-57 (2005).

19. Ahlskog JE. Parkinson's disease treatment guide for physicians. Oxford University Press, USA (2009).

20. Navarro Sanchez L. Genetics and epigenetics in Parkinson's disease (2014).

21. DeLong MR, Georgopoulos AP. Motor functions of the basal ganglia. Compre Physiol (2011).
22. Samantaray S, Mohanakumar KP. Involvement of calcium in 1-methyl-4-phenyl-1, 2, 3, 6-tetrahydropyridine (MPTP)induced experimental parkinsonism. 106: (2003).

23. Chao MV. Neurotrophins and their receptors: a convergence point for many signalling pathways. Nat Rev Neurosci 4: 299309 (2003).

24. Jain KK. Neuroprotection in traumatic brain injury. In The Handbook of Neuroprotection (Humana Press) 217-253 (2011).

25. Hadaczek P, Wu G, Sharma N, et al. GDNF signaling implemented by GM1 ganglioside; failure in Parkinson's disease and GM1-deficient murine model. Exp neurol 263: 177-189 (2015).

26. Siegel GJ, Chauhan NB. Neurotrophic factors in Alzheimer's and Parkinson's disease brain. Brain Res Rev 33: 199-227 (2000).

27. Blum D, Torch S, Lambeng N. Molecular pathways involved in the neurotoxicity of 6-OHDA, dopamine and MPTP: contribution to the apoptotic theory in Parkinson's disease. Progress in neurobiol 65: 135-172 (2001).

28. Connor B, Dragunow M. The role of neuronal growth factors in neurodegenerative disorders of the human brain. Brain Res Rev 27: 1-39 (1998).

29. Martins IJ. Early diagnosis of neuron mitochondrial dysfunction may reverse global metabolic and neurodegenerative disease. Glob J Med Res 2: 1-8 (2016).

30. de Tassigny XDA, Pascual A, Lopez-Barneo J. GDNF-based therapies, GDNF-producing interneurons, and trophic support of the dopaminergic nigrostriatal pathway. Implications for Parkinson's disease. Front in neuroana 9 (2015).

31. Kirik D, Rosenblad C, Björklund A Mandel RJ. Long-term rAAV-mediated gene transfer of GDNF in the rat Parkinson's model: intrastriatal but not intranigral transduction promotes functional regeneration in the lesioned nigrostriatal system. $J$ Neurosci 20: 4686-4700 (2000).

32. Kearns CM, Gash DM. GDNF protects nigral dopamine neurons against 6-hydroxydopamine in vivo. Brain res 672: 104111 (1995).

33. Rosenblad C, Kirik D, Björklund A. Sequential administration of GDNF into the substantia nigra and striatum promotes dopamine neuron survival and axonal sprouting but not striatal reinnervation or functional recovery in the partial 6-OHDA lesion model. Exp neurol 161: 503-516 (2000).

34. Lindholm P, Saarma M. Novel CDNF/MANF family of neurotrophic factors. Develop neurobiol 70: 360-371 (2010).

35. Airaksinen MS, Saarma M. The GDNF family: signalling, biological functions and therapeutic value. Nat Rev Neurosci 3: 383-394 (2002).

36. Planken A. Role of GDNF and its Cross-Talk with Other Growth Factors in the Dopaminergic System (2012).

37. Lotharius J, Brundin P. Pathogenesis of Parkinson's disease: dopamine, vesicles and $\alpha$-synuclein. Nat Rev Neurosci 3: 932942 (2002).

38. Agnati LF, Baldelli E, Andreoli N. On the key role played by altered protein conformation in Parkinson's disease. J Neural Trans 115: 1285-1299 (2008).

39. Meka DP, Müller-Rischart AK, Nidadavolu P. Parkin cooperates with GDNF/RET signaling to prevent dopaminergic neuron degeneration. J clin inves 125: 1873 (2015). 
40. Klein P. Functions of GDNF/Ret signaling in models of autosomal recessive Parkinson's disease (Doctoral dissertation 2011).

41. Kramer ER, Liss B. GDNF-Ret signaling in midbrain dopaminergic neurons and its implication for Parkinson disease. FEBS letters 589: 3760-3772 (2015)

42. Shulman LM, Taback RL, Rabinstein AA. Non-recognition of depression and other non-motor symptoms in Parkinson's disease. Parkin rel dis 8: 193-197 (2002).

43. Pierucci M, Galati S, Valentino M. Nitric oxide modulation of the basal ganglia circuitry: therapeutic implication for Parkinson's disease and other motor disorders. CNS \& Neurological Disorders-Drug (2011).

44. Kramer ER, Aron L, Ramakers GM. Absence of Ret signaling in mice causes progressive and late degeneration of the nigrostriatal system. PLoS biol 5: 39 (2007).
45. Aron L, Klein R. Repairing the parkinsonian brain with neurotrophic factors. Trend neurosci 34: 88-100 (2011).

46. Choudhury A, Rao VD, Varma RJ, Banerjee TS, Kumar RA, Adapa D. Cognitive and Psychological Anomalies in Parkinson's Disease: An Insight into Non-Motor Characteristic Features. Neuropsy (Lond) 7: 1069-1080 (2017).

47. Deshmukh R, Sharma V, Mehan S, Sharma N, Bedi KL. Amelioration of intracerebroventricular streptozotocin induced cognitive dysfunction and oxidative stress by vinpocetine-a PDE1 inhibitor. Eur J pharmacol 620: 49-56 (2009).

48. Datta S, Jamwal S, Deshmukh R, Kumar P. Beneficial effects of lycopene against haloperidol induced orofacial dyskinesia in rats: Possible neurotransmitters and neuroinflammation modulation. Eur J pharmacol 771: 229-235 (2016). 